\title{
Dose, timing, schedule, and the choice of targeted epitope alter the efficacy of anti-CD22 immunotherapy in mice bearing human lymphoma xenografts
}

\author{
Robert T. O'Donnell $\cdot$ Yunpeng Ma • \\ Hayes C. McKnight · David Pearson • \\ Joseph M. Tuscano
}

Received: 16 November 2008/Accepted: 16 April 2009/Published online: 13 May 2009

(C) The Author(s) 2009. This article is published with open access at Springerlink.com

\begin{abstract}
CD22 is a cell-surface adhesion molecule on most B-cell NHL, so it is a promising target for immunotherapy. HB22.7 is an anti-CD22 mAb that binds the two $\mathrm{NH}_{2}$-terminal immunoglobulin domains and specifically blocks the interaction of CD22 with its ligand. CD22blocking mAbs induce apoptosis in neoplastic B-cells and are functionally distinguishable from other anti-CD22 mAbs. This study assessed the optimal dose, route, schedule, and the targeted CD22 epitope. Raji NHL-bearing nude mice were studied. A non-blocking anti-CD22 mAb (HB22.27) was used as a control. HB22.27 had minimal effect, whereas HB22.7 improved survival and shrank tumors substantially. HB22.7 doses greater than $1.4 \mathrm{mg} /$ week did not further increase efficacy (or toxicity). Tumors less than $200 \mathrm{~mm}^{3}$ had a higher response rate than did larger tumors. Various schedules of HB22.7 administration were tested; one dose every other week was more effective than more or less frequent dosing. Pharmacokinetic studies revealed that the half-life of HB22.7 was 28 days; this correlated with the time needed to re-populate cell-surface CD22 after treatment with HB22.7. Immuno-PET showed that NHL was rapidly and specifically targeted by copper64-labeled-HB22.7. This study provided data as to an
\end{abstract}

R. T. O'Donnell · Y. Ma · H. C. McKnight · D. Pearson · J. M. Tuscano

Division of Hematology and Oncology, Department of Internal Medicine, University of California, Davis, Cancer Center, 4501 X Street, Suite 3016, Sacramento, CA 95817, USA

J. M. Tuscano

e-mail: joseph.tuscano@ucdmc.ucdavis.edu

R. T. O'Donnell ( $\square)$

Veteran's Administration Northern California Healthcare

System, Mather, CA, USA

e-mail: robert.odonnell@ucdmc.ucdavis.edu optimal dose, route, schedule and interval between doses of HB22.7.

Keywords CD22 $\cdot$ Lymphoma $\cdot$ Antibody

\section{Introduction}

NHL is the sixth most common cause of cancer-related deaths in the United States. Standard therapy relies on toxic drugs which, while initially effective, are curative in less than $50 \%$ of cases. Rituximab, a chimeric anti-CD20 mAb has become integral to many drug regimens for NHL therapy [1]. Because of the safety and efficacy of targeted $\mathrm{mAb}$ like rituximab, new antibodies are being tested to see if they may also be effective, synergistic with rituximab, or useful when the NHL becomes resistant to other therapies.

CD22 is a B-lymphocyte-specific glycoprotein and adhesion molecule found on the outer surface membrane of nearly all mature B-cells. Most NHL express CD22 as well, so $\mathrm{CD} 22$ is a promising target for immunotherapy [2, 3]. CD22 is a member of the immunoglobulin superfamily, with an extracytoplasmic component that contains seven immunoglobulin-like domains. The two amino-terminal domains of CD22 mediate adhesion to sialic acid bearing ligands and can bind multiple hematopoietic cell types [4-6].

A panel of anti-CD22 mAbs that targets CD22 was evaluated [6]. Few studies have examined the clinical or pre-clinical effects of $\mathrm{mAb}$ binding to different epitopes of the same receptor. Anti-CD22 mAbs that bind the two $\mathrm{NH}_{2}$-terminal immunoglobulin domains of CD22 and specifically block the interaction of CD22 with its ligand were identified. These "blocking" mAbs induce apoptosis in neoplastic B-cells [4]. Studies that eliminated the first 
two domains of $\mathrm{CD} 22$ demonstrated that the in vivo binding of these domains to their ligands was critical for B-cell survival [7]. By contrast, mAbs that do not block ligand binding have only modest functional effects $[8,9]$. Ligand blocking anti-CD22 mAbs (e.g. HB22.7) are unique and functionally distinguishable from other anti-B-cell, and even other anti-CD22 mAb. Consistent with this data, the blocking anti-CD22 mAbs are more effective than nonblocking $\mathrm{mAb}$ at initiating CD22-mediated signal transduction in general, and the stress activated protein kinase (SAPK) pathway specifically $[5,8,9]$. We characterized several anti-CD22 mAbs that have unique signaling properties, pro-apoptotic effects, and lymphomacidal properties; based on these properties HB22.7 was chosen for further development [10]. HB22.7 has recently been humanized (hHB22.7) and has the potential to become an exciting, new treatment for NHL.

Other effective $\mathrm{mAb}$ have entered the clinical realm without much preclinical data to show what may be the most effective dose, interval between doses, route of administration, or the effect of other critical factors such as tumor size. In addition, other anti-CD22 mAbs have been evaluated in human clinical trials and demonstrated only modest efficacy [11]. These anti-CD22 mAbs bind to a different epitope than does HB22.7, do not block ligand binding, and do not have functional effects [12]. The goal of this study is to characterize the dose, schedule, CD22 epitope, and tumor related factors that can guide the translation of the hHB22.7 toward a human clinical trial.

\section{Methods}

\section{Cell culture}

The Raji human NHL cell line (ATCC) was maintained in RPMI (GIBCO, Gaithersburg, MD) with 10\% v/v fetal bovine serum (FBS) (Cambrex, Baltimore, MD) supplemented with glutamine, penicillin and streptomycin. For the in vitro studies, cells were cultured in $225 \mathrm{~cm}$ filter vented flasks. Cells were maintained in a humidified tissue culture incubator at $37^{\circ} \mathrm{C}$ with $5 \% \mathrm{CO}_{2}$ split every $2-3$ days.

Monoclonal antibodies

HB22.7 and HB22.27 were purified from ascites (Covance, Princeton, NJ) and have been previously characterized [6].

\section{HB22.7 pharmacokinetics}

A FACS-based assay was used to serially measure the concentration of HB22.7 in the serum of mice bearing Raji xenografts after a single intravenous (IV) dose (1.4 mg).
To establish a standard curve, mouse serum with known concentrations of HB22.7 were used for FACS analysis of the Raji cell line. Concentrations of directly FITC-conjugated HB22.7 were plotted against mean fluorescence intensity (MFI) compensating for background effects of unstained Raji cells and mouse serum. A linear relationship was observed between 0.2 and $2.0 \mu \mathrm{g}$ HB22.7/cc and MFIs between 100 and 600. To avoid the potential confounding effects of $\mathrm{CD} 22$ saturation, the MFIs within this range were used to calculate the serum concentration of HB22.7 after treatment. Serum samples were obtained from the tail vein at baseline and at indicated intervals after treatment.

\section{Mouse experiments}

Nude mice bearing human xenografts from the Raji NHL cell line has proven utility for evaluating the efficacy of new treatments [10]. To prevent rejection and ensure tumor implantation, $n u / n u$ mice on a Balb/c background (Harland Laboratories, Indianapolis, IN) received whole body irradiation (400 rad) with a $6 \mathrm{MeV}$ beam from the linear accelerator. Radiation before xenograft implantation was shown to be a useful technique in this model and has been used in all our prior murine studies. After 3 days, mice were injected subcutaneously with 3-6 $\times 10^{6}$ Raji NHL cells in media $(100 \mu \mathrm{l})$. In $2-3$ weeks, tumors reached an acceptable size for treatment assessment $\left(20-300 \mathrm{~mm}^{3}\right)$ [41, 42]. In some experiments, treatment with the $\mathrm{mAb}$ was done 1 day after the xenograft was implanted rather than in established tumors. All mice were injected with mAb IV though the tail vein except for the immuno-positron emission tomography (iPET) experiment. In the iPET experiments, mice were also injected subcutaneously (SQ) or intraperitoneally (IP).

Mice were assessed for toxicity by twice-weekly measurement of their weight, activity, and blood counts for the first 28 days, then weekly for the rest of the 84-day study period (standard assessment of toxicity by the UC Davis School of Veterinary Medicine Lab Animal Clinic). Tumor diameter was assessed in three dimensions using calipers and the volume calculated by the ellipsoid volume equation $(\mathrm{d} 1 \times \mathrm{d} 2 \times \mathrm{d} 3 \times 0.52=$ ellipsoid volume $)$. Tumoricidal effects were assessed by twice weekly tumor volume measurement. Tumor responses will be categorized as follows: cure (C, tumor disappeared and did not re-grow by the end of the 84-day study); complete regression (CR, tumor disappeared for at least 7 days but later re-grew); partial regression (PR, tumor volume decreased by $50 \%$ or more for at least 7 days then re-grew).

Statistical analysis

Differences in response among treatment groups were evaluated using the Kruskal Wallis rank sum test. Survival 
time was also evaluated using the Kruskal Wallis test. If an animal was sacrificed due to tumor-related causes, the last volume was carried forward and used in the analysis of later time points. Analysis of variance was used to test for differences among treatment groups. $P$ values are twotailed and represent the nominal $P$ values. Protection for multiple comparisons is provided by testing only within subsets of groups found to be statistically significantly different.

\section{I-PET}

Copper-64 labeled HB22.7 was used to determine the ability of HB22.7 to specifically target NHL in vivo [13]. ${ }^{64} \mathrm{Cu}$ (a positron emitter) combines all three modes of decay: electron capture $(41 \%)$, beta - $(40 \%)$ and beta $+(19 \%)$ making it a useful radionuclide for both imaging and therapy. ${ }^{64} \mathrm{Cu}$ was produced on the biomedical cyclotron at Washington University and supplied as ${ }^{64} \mathrm{CuCl}_{2}(0.1 \mathrm{M} \mathrm{HCl})$. The bifunctional chelating agent, DOTA $(1,4,7,10$-tetraazacyclododecane $N, N^{\prime}, N^{\prime \prime}, N^{\prime \prime \prime}$-tetraacetic acid (DOTA) contains a reactive functionality to form a covalent attachment to proteins and a strong metal-binding group to chelate radiometals [14]. DOTA-HB22.7 was prepared by incubation with DOTANHS-ester at pH 5.5. DOTA-HB22.7 was labeled with ${ }^{64} \mathrm{Cu}-$ acetate in $0.1 \mathrm{M}$ ammonium acetate, $\mathrm{pH}$ 5.5. After incubation $1 \mathrm{mM}$ EDTA terminated the reaction. HPLC purification was then performed to purify the ${ }^{64} \mathrm{Cu}$-DOTA-HB22.7.

PET scanning was performed using a microPET II small-animal scanner $[15,16]$, a system that reconstructs images with a spatial resolution of approximately $1.2 \mathrm{~mm}$ using conventional analytic reconstruction algorithms. The resolution is quite isotropic at the center of the field of view, resulting in a resolution volume of $1.7 \mathrm{~mm}^{3}$. The absolute sensitivity of the scanner at the center of the field of view is $2.25 \%$ using the default energy window settings of $250-750 \mathrm{keV}$ and a coincidence timing window of $10 \mathrm{~ns}$. The imaging field of view of the scanner is $10 \mathrm{~cm}$ in the transverse direction and $4.8 \mathrm{~cm}$ in the axial direction. High quality images were obtained using injected doses of $100 \mu \mathrm{Ci}$ and imaging times of 5-10 min.

Mice were anesthetized with isoflurane induction and maintenance during imaging and monitored visually throughout the study. A scavenging system trapped exhaled anesthetic gas. Mice were injected with approximately $200 \mu \mathrm{Ci}$ (range $198-216 \mu \mathrm{Ci}$ ) of ${ }^{64} \mathrm{Cu}-\mathrm{DOTA}-\mathrm{HB} 22.7$ in $160 \mu \mathrm{l}$ saline by one of three routes: IV via the tail vein, IP on the right flank, or SQ between the shoulders $(n=3$ per route of administration). The activity in the syringe before and after injection was measured in a dose calibrator and decay-corrected so that the injected dose was known. At the moment of radiotracer injection, data acquisition was initiated in list mode on the microPET II scanner. Imaging continued for $90 \mathrm{~min}$ or until sufficient counts were recorded. Then, the list mode data was binned into time frames and reconstructed with a statistical 3D reconstruction algorithm [16]. Absolute quantification was achieved by calibrating the mouse images with the image of a cylinder containing a uniform concentration of positronemitting radionuclide with a similar geometry and volume as a mouse. The calibration scan was acquired under identical conditions and reconstruction parameters as the mouse scans and has similar attenuation and scatter characteristics. Quantification with this method, without scatter and attenuation correction was better than $10 \%$ when comparing contralateral sites in the same mouse or the same site across different mice of similar weight. Image analysis was carried out using ASIPro software and in-house IDL-based tool for defining and quantifying uptake in volumes of interest.

\section{Results}

In previous experiments that established the preclinical efficacy of HB22.7, a single dose of HB22.7 (1.4 mg) was administered IV [10]. In the current study, the $1.4 \mathrm{mg}$ dose was compared to higher single HB22.7 doses to see if an even better response could be obtained, Fig. 1. The choice of $1.4 \mathrm{mg}$ was based on the equivalent dose that effectively induced apoptosis in vitro, the equivalent dose of rituximab (with dosage adjustments for the increased surface area in mice as compared to humans), and the dose previously shown to have efficacy in mice bearing Raji xenografts. Although the single $4.2 \mathrm{mg}$ dose produced a slightly lower peak tumor volume than did the 1.4 or $2.8 \mathrm{mg}$ doses, the results of treatment with any single dose of HB22.7 were similar to each other but greatly different than the pattern of disease progression seen in the untreated controls. Figure 1 showed that $1.4 \mathrm{mg}$ of HB22.7 was sufficient to cause tumor shrinkage and that higher doses were no more effective. A one-way ANOVA showed that all HB22.7 treatment doses resulted in significant tumor shrinkage when compared to the control $P<0.001$; none of the HB22.7 doses were significantly different from each other, $P>0.05$. As is characteristic of this model, some untreated mice had spontaneous partial responses. For Fig. 1, 26\% of untreated mice had spontaneous PR, but the RR for mice treated with only one dose of HB22.7 was 53\% and 7\% of the responses were complete.

Multiple studies were performed using HB22.7 to treat nude mice bearing Raji xenografts. Similar to what is seen after treatment with rituximab, it was observed that some mice had CRs but some mice did not respond at all. One factor that related to the response and survival was the size of the initial tumor, Fig. 2. HB22.7 was much more 


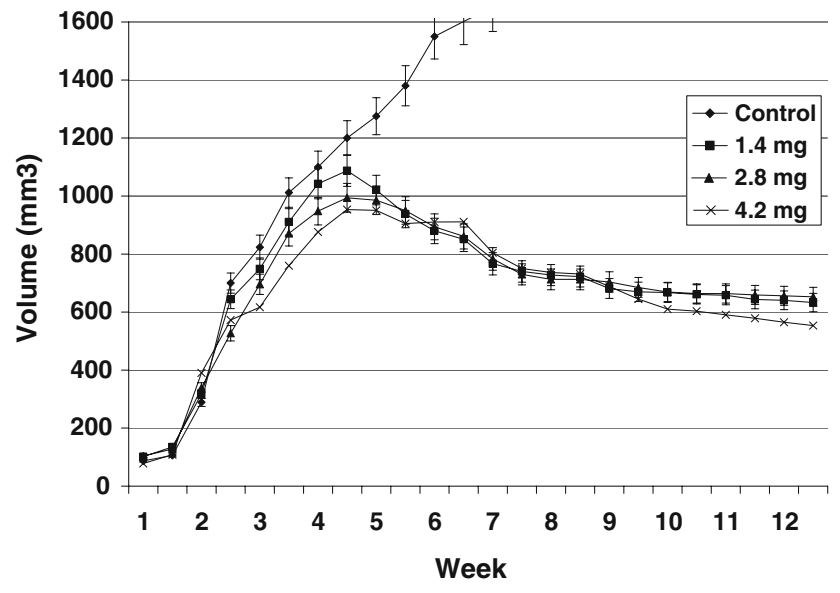

Fig. 1 HB22.7 immunotherapy resulted in a decrease in tumor volume in nude mice bearing Raji-NHL xenografts (10 mice/group). Each dose cohort $(1.4,2.8,4.2 \mathrm{mg}$ ) had efficacy showing that $1.4 \mathrm{mg}$ was a reasonable dose for subsequent experiments

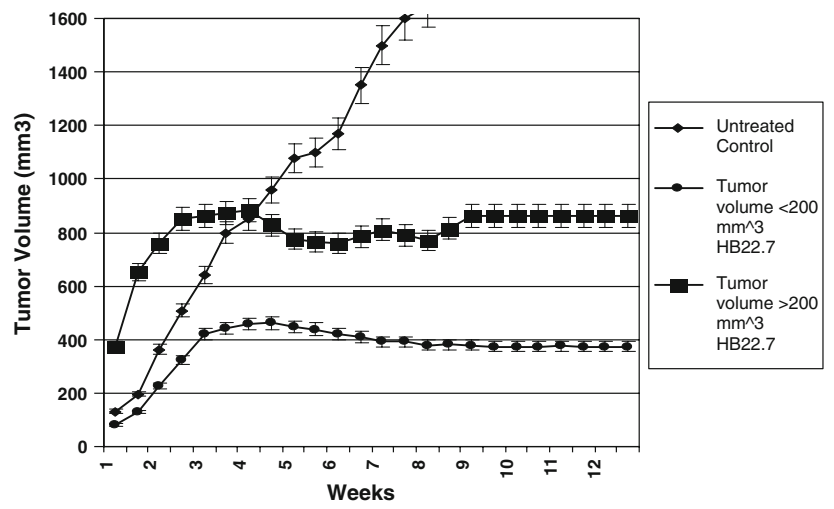

Fig. 2 The effect of starting tumor volume on the efficacy of HB22.7. Treatment of tumors that were initially large $\left(>200 \mathrm{~mm}^{3}\right)$ was compared with treatment of smaller tumors $\left(<200 \mathrm{~mm}^{3}\right)$. In the treatment group having smaller tumors, there was more tumor shrinkage when compared to tumors that were initially $>200 \mathrm{~mm}^{3}$. Control mice $n=20$; HB22.7-treated mice $>200 \mathrm{~mm}^{3}, n=5$; HB22.7-treated mice $<200 \mathrm{~mm}^{3}, n=21$

effective when the initial size of the tumor was less than $200 \mathrm{~mm}^{3}$. The CR rate, tumor volume reduction, and survival were all better when tumors were less than $200 \mathrm{~mm}^{3}$ at the time of HB22.7 administration. In this experiment, untreated controls had a spontaneous remission rate of 5\% (one PR). Mice with tumors less than $200 \mathrm{~mm}^{3}$ when treated with one dose of HB22.7 had a 48\% RR (33\% CRs and $15 \%$ PRs), in addition to flattening of the tumor growth rate. Mice with tumors greater than $200 \mathrm{~mm}^{3}$, when treated with one dose of HB22.7 had no shrinkage that qualified for even a PR (although their growth rate slowed). The difference between response rates for small tumors versus untreated controls was significant $(P=0.012)$, however, there was no significant difference between the response rate of untreated controls and mice bearing xenografts
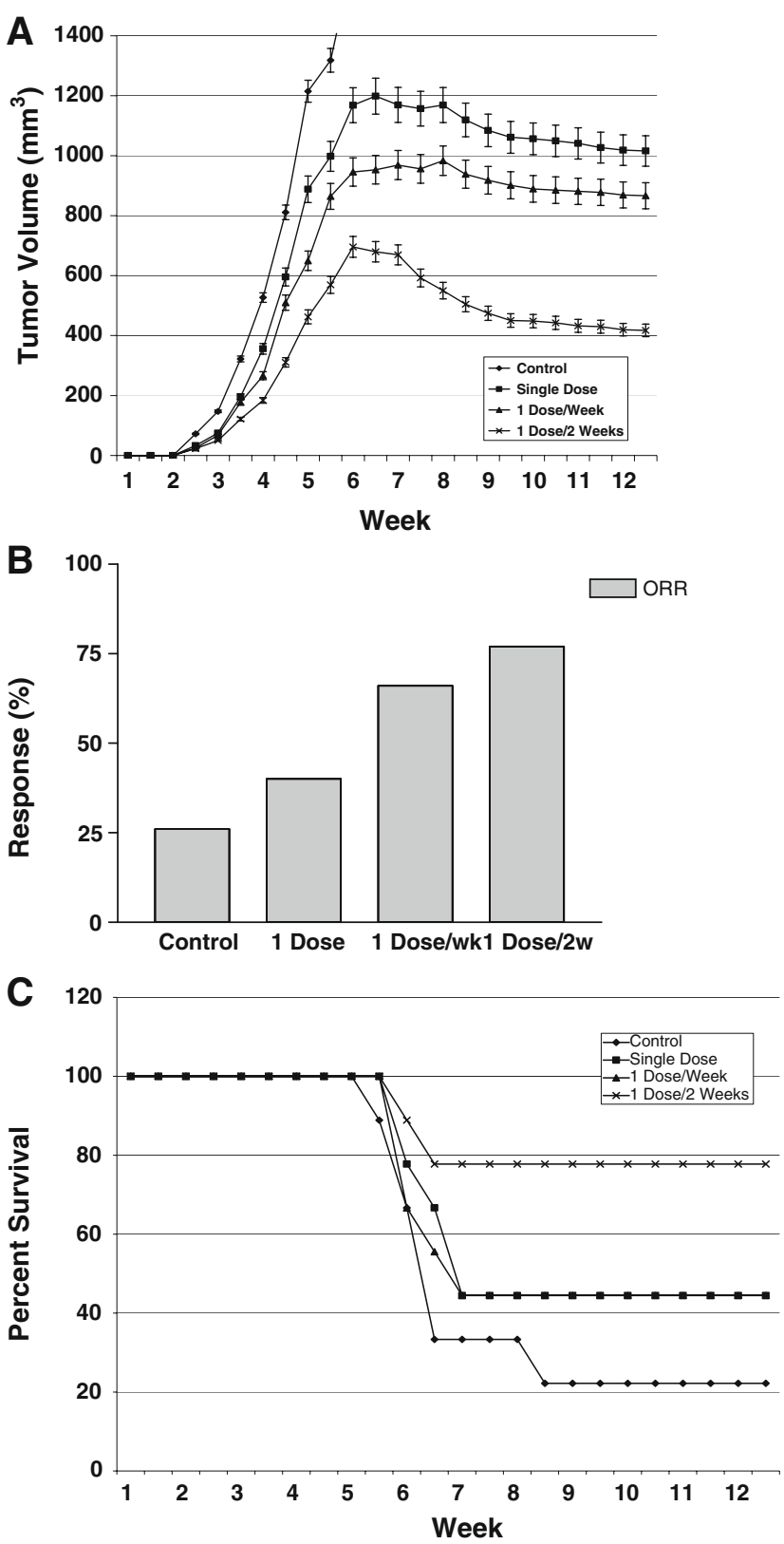

Fig. 3 The effect of dosing schedule on tumor response rate. Groups of nine mice were treated with HB22.7 before Raji NHL tumors had become established. HB22.7 (1.4 mg) was administered on: Day 1, Day 1 and weekly for 6 weeks, Day 1 and every other week for 12 weeks, or were untreated. Mice were monitored for tumor volume (a), response rate (b) and survival (c)

greater than $200 \mathrm{~mm}^{3}$. Similarly, the difference in CRs between the small treated xenografts and untreated controls was $P=0.015$.

The concept of treating relatively low-volume tumors was tested further. Mice were treated with HB22.7 $(1.4 \mathrm{mg}) 1$ day after the implantation of the Raji cells; mice were followed for development of a tumor, its growth kinetics, and survival, Fig. 3. Early treatment (low tumor 
volume, non-established tumors) was at least as effective as the same dose and schedule of HB22.7 given to mice with small established tumors, Fig. 2. The CR rate for all mice treated with HB22.7 in this experiment was 9/27 (33\%), similar to mice with small established tumors that were treated with HB22.7, see Fig. 2.

Most therapies for NHL are given more than one time. There is no prior data to assess the response rate produced by HB22.7 in a mouse model when it is given in multiple doses and with differing schedules of administration. Figure 3 shows that multiple doses result in more tumor shrinkage than did one dose. Thirty-seven percent of mice treated with more than one dose had a CR, whereas $11 \%$ of mice treated with one dose had a CR; none of the untreated control mice had a spontaneous CR. For the response rate data, the $P$ values for administration of HB22.7 weekly versus the untreated control, and once every other week versus the untreated control, were 0.043 and 0.011 , respectively. All other comparisons did not reach statistical significance, although the $P$ value for one single administration of HB22.7 versus the control was 0.06. However, the interval between doses was also important as indicated by the better tumor shrinkage when HB22.7 was given every other week for six doses, compared to weekly administration of HB22.7 for 6 consecutive weeks, Fig. 6 a. The survival of mice treated every other week with HB22.7 was significantly better than the untreated control (Fig. 3c), and the response rate was higher for mice treated every other week as well. For survival, the only significantly different comparison was for HB22.7 administration once every other week versus the untreated control; $P=0.026$. The best interval between doses studied was 2 weeks.

It has been well described that the binding of CD22 by $\mathrm{mAb}$ mediates internalization; $\mathrm{CD} 22$ reappearance at the cell surface does not occur until the levels of anti-CD22 nadir $[12,17]$. To determine if the in vivo HB22.7 half-life and the xenograft's CD22 cell surface expression were related, a FACS-based assay to measure HB22.7 levels was used. This revealed that after a single $1.4 \mathrm{mg}$ dose, HB22.7 did not go below $0.5 \mathrm{ug} / \mathrm{ml}$ for 14 days and below $0.4 \mathrm{ug} / \mathrm{ml}$ for nearly 30 days. The measured half-life of HB22.7 was $28 \mathrm{~h}$, Fig. 4. To assess for changes in CD22 surface expression in the NHL xenografts after treatment, fine needle aspirations (FNA) were done serially; the samples were examined by flow cytometry for CD22 surface expression. This analysis revealed that CD22 became internalized quickly, within $6 \mathrm{~h}$ of treatment and did not return to baseline surface expression levels for 14 days, Fig. 5.

Studies that examine the clinical effects of specific CD22 epitope binding have not been done previously. HB22.7 binds to domain 2 of CD22 and blocks ligand binding [4-6]. We conducted a xenograft study comparing the efficacy of HB22.7 to that of HB22.27 (which binds to

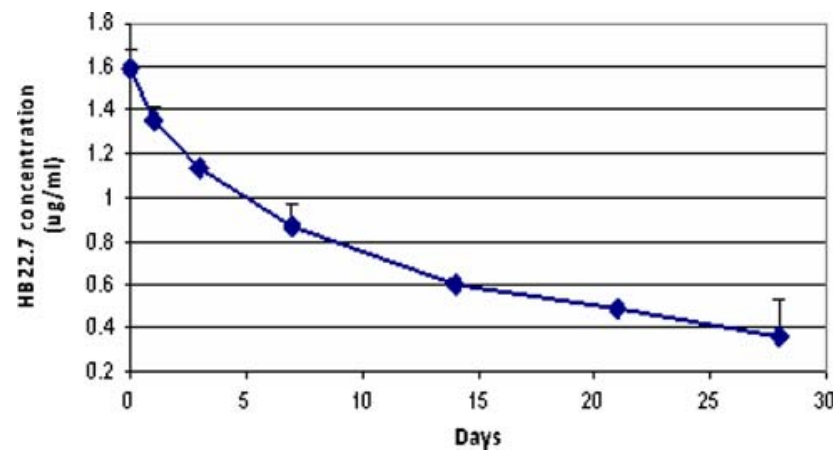

Fig. 4 HB22.7 half-life in mice bearing Raji xenografts. FACS was used to measure the concentration of HB22.7 after administration of a single $1.4 \mathrm{mg}$ dose. The analysis was repeated three times; error bars represent the standard deviation

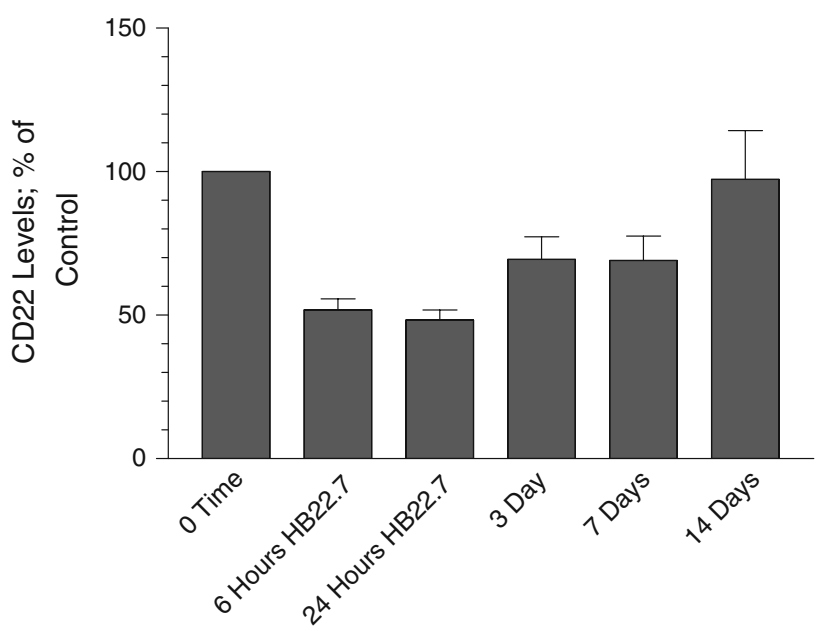

Fig. 5 CD22 surface expression in mice bearing Raji xenografts. Serial FNA of Raji xenografts were used to assess CD22 levels after treatment with a single dose of HB22.7. The experiment was repeated three times with the error bars representing the standard deviation

domain 3) [6]. Figure 6 shows that the non-blocking antiCD22 mAb (HB22.27) did not result in as much tumor shrinkage as did the blocking anti-CD22 mAb, HB22.7. The overall response rate for mice treated with HB22.7 was $84 \%$; the overall response rate for the mice treated with HB22.27 was $41 \%, P<0.0001$. There were twice as many PRs and twice as many CRs in the HB22.7 group than in mice treated with HB22.27. This translated into improved overall survival with $85 \%$ alive at the end of the 12 week study after treatment with HB22.7 versus $42 \%$ after treatment with HB22.27.

Previous studies demonstrated excellent targeting of Raji xenografts by indium-111-DOTA-HB22.7 after IV injection [10]. However, micro-PET scanning offers a greater resolution and sensitivity than gamma camera imaging of indium-111 and iPET allows for real-time in vivo targeting and biodistribution studies [18-20], therefore we tested ${ }^{64} \mathrm{Cu}$-DOTA-HB22.7. Many experimental 

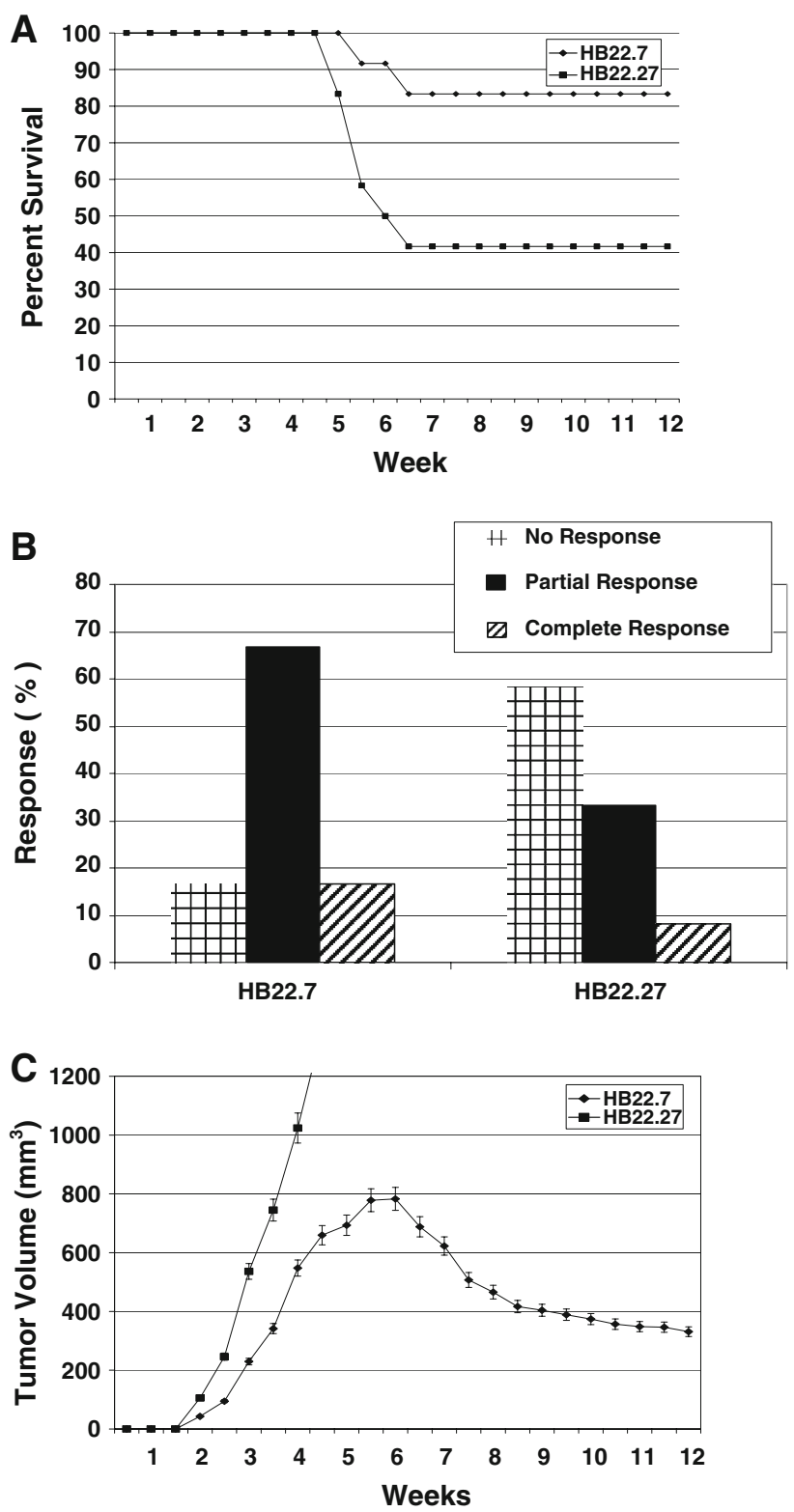

Fig. 6 Effect of blocking versus non-blocking anti-CD22 antibodies on response. Treatment with the blocking anti-CD22 mAb HB22.7 was effective, whereas the control non-blocking anti-CD22 mAb HB22.27 was ineffective. Twelve mice were in each group. Survival (a) and response rate (b) were better in mice treated with HB22.7. The average reduction in tumor volume (c) was far better in mice treated with HB22.7 versus HB22.27

agents are administered IV via the tail vein; this can be difficult and irreproducible, especially after repeated injections in the same animal for longitudinal studies. Easier and more reproducible methods of administration would be useful provided that tumor targeting and biodistribution are not adversely altered. There are no studies that compare targeting after different routes of anti-CD22 radiopharmaceutical administration. Using copper-64 as a positron emitter it was shown by iPET scanning with
${ }^{64} \mathrm{Cu}$-DOTA-HB22.7 that HB22.7 could target Raji-NHL using the usual route (IV), but also by SQ or IP injection, Fig. 7. These images show that each route of administration resulted in excellent tumor targeting by $48 \mathrm{~h}$ after injection. The background was low indicating good specificity and there was non-specific targeting of non-NHL tissue.

\section{Discussion}

Recent studies have examined the role of the CD22 ligand binding domain in normal and malignant B-cell survival. Murine models were used to demonstrate that the CD22 ligand binding domain is critical for normal B-cell survival. It was conclusively shown that mAbs that block CD22 ligand binding inhibit survival in vivo. Mouse antimouse CD22 ligand blocking mAbs that eradicated lymphoma independent of complement and $\mathrm{F}_{\mathrm{c}}$ receptor-bearing effector cells have been developed [21, 22].

This study examined the optimal dose, interval, and route of administration of HB22.7. In addition, the effects of initial tumor volume and CD22 epitope binding were examined. A safe and effective dose of HB22.7 was $1.4 \mathrm{mg}$; above that dose further improvement in efficacy was not demonstrable. However, this could change with variations in tumor burden and dosing intervals. Recent patient-specific dosing studies with rituximab have confirmed variable serum levels in patients likely due to their differing tumor burdens and antigen densities [23]. A dose escalation study is still needed to determine the optimal dose in humans, however, this study provides good insight into the appropriate starting dose for human clinical trials.

There are two distinct ways to initiate treatment in this model. First, treatment can be done on established tumors. This method has the advantage of having measurable disease and knowing that the tumor did in fact grow in a particular mouse. The downside of this choice is that we conclusively showed that larger tumors have a lower response rate so small differences in the tumor size may obscure the results. It is important to note, but not unexpected, that larger tumors do not respond well. These data suggest that pretreatment with other agents to reduce tumor bulk prior to treatment with anti-CD22 mAb would be a valid strategy. Also, since the tumors are already large and growing when treatment is initiated, there is less time for the therapy to work before the mice need to be euthanized. The other way to initiate treatment is to give the first dose of $\mathrm{mAb}$ on the day after the NHL cells have been implanted to form the xenograft. At this point, all mice will have been injected with the same number of cells so their starting point is more homogeneous as the implanted cells have not had a chance to grow at different rates, or to different sizes. Because the xenograft is then at its smallest, the mAb will 

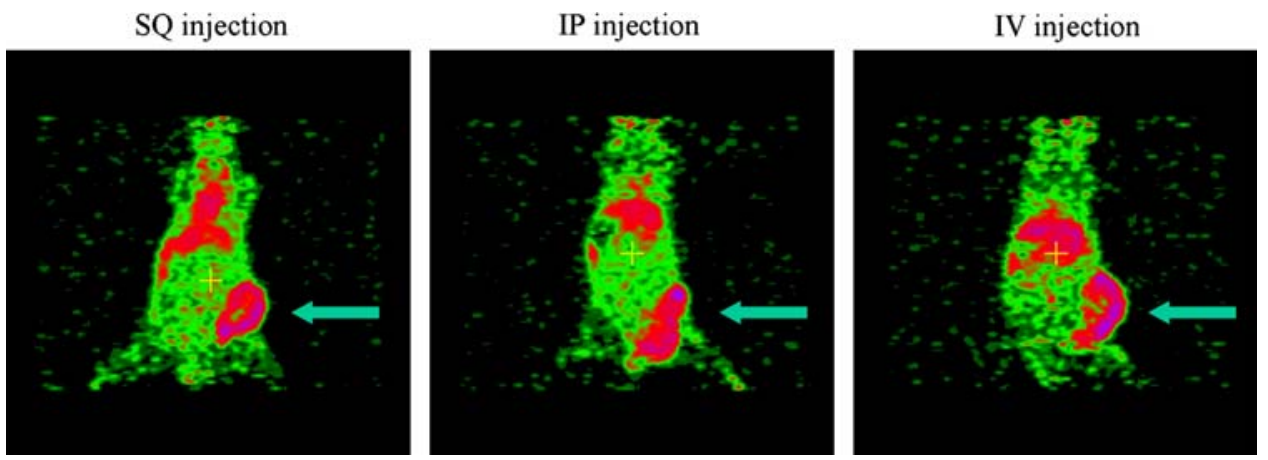

Fig. 7 In vivo iPET images: ${ }^{64} \mathrm{Cu}$-DOTA-HB22.7 targets Raji xenografts at $48 \mathrm{~h}$ after injection. Xenograft bearing mice were injected IV, IP, or SQ with approximately $200 \mu \mathrm{Ci}$ of ${ }^{64} \mathrm{Cu}$-DOTA-
HB22.7. Mice were imaged using a micro-PET II at $48 \mathrm{~h}$ after injection. $n=3$ mice per injection route. Xenograft locations are indicated by arrows. a IV injection. b IP injection. c SQ injection be able to target it optimally. We demonstrated that differences between experimental groups and the controls were easier to detect when the mAb therapy was given on the day after xenograft implantation rather than on established tumor. This mimics treatment of non-bulky tumors or minimal residual disease. In some ways, it is not surprising to find that smaller tumors have a better response rate than large ones, and that tumors that are found early (the analogy for treating the mice 1 day after xenograft implantation) are easier to treat than those that are well established. However, this is indeed a relevant topic that directly relates to humans receiving immunotherapy. For example, a mAb that is efficacious in low tumor-volume settings may have an important role in clearing the bone marrow of residual malignant cells. Another role could be related to eliminating foci of NHL in association with stem cell harvest or bone marrow transplantation. Clinical trials will be needed to further explore this topic in patients.

Previous studies with epratuzumab showed that nearly $80 \%$ of CD22 was internalized due to prolonged $\mathrm{mAb}$ binding [12]. Based on this finding, we hypothesized that closely spaced doses would not significantly improve efficacy because of "loss of target" (CD22) due to its internalization. Increasing the interval between doses may allow for return of CD22 surface expression. In this study, giving six doses of HB22.7 every other week was the most effective schedule; better than six consecutive weekly doses. We demonstrated that the CD22 surface expression on the implanted Raji xenografts was lost within $6 \mathrm{~h}$ of treatment and did not return for nearly 14 days. This correlated with a prolonged HB22.7 half-life and validates our hypothesis that receptor saturation by $\mathrm{HB} 22.7$ results in a loss of target and diminished efficacy. This is likely because the target of HB22.7 (CD22) was saturated by weekly doses mediating CD22 internalization, thus making weekly dosing irrelevant. Six doses of HB22.7 every other week likely results in a more prolonged therapeutic level of
HB22.7 when the target CD22 is present leading to improved efficacy.

The iPET experiments demonstrated that radiolabeled HB22.7 would specifically target NHL in vivo with low background (non-specific binding). This data is useful in that it showed that the SQ and IP doses, which are easier and more reproducible to administer to mice than is IV, were a valid experimental technique. While the vast majority of mAb-based therapeutics are administered IV, some have been administered SQ [24] without data assessing the equivalency of targeting and pharmacokinetics. Thus, this data may facilitate the translation of humanized HB22.7 to human clinical trials.

The control antibody, HB22.27 binds CD22 as well as HB22.7 does; however, they bind different epitopes. Our previous studies show that binding to the correct epitope (as does HB22.7) initiates a signaling cascade that can cause apoptosis in NHL cells [8,9]. As well, other studies have shown that the first two domains of CD22 are critical for B-cell survival [7] and mouse derived anti-mouse, antiCD22 mAbs that are directed at CD22 ligand blocking domains eradicate murine NHL [23]. Data presented here show that there is a great difference in efficacy when the appropriate CD22 epitope is targeted. Other anti-CD22 $\mathrm{mAb}$ have been studied and have not generated significant responses in animal models or human clinical trials [11, 12] likely because they, like HB22.27 bind to a non-ligand blocking region of $\mathrm{CD} 22$. This is one of the first studies that clearly shows that the specific epitope bound by a mAb effects efficacy.

This study showed that $1.4 \mathrm{mg}$ of $\mathrm{HB} 22.7$ was an appropriate dose upon which to base further clinical studies. Treatment of small and/or early tumors resulted in better efficacy than did treatment of large established tumors. An every other week schedule of HB22.7 administration was sufficient for therapy. This schedule is effective likely due to rapid CD22 internalization without 
return of $\mathrm{CD} 22$ to the cell surface for nearly 2 weeks due to persisting HB22.7 levels. HB22.7 targets tumor equally well by IV, SQ or IP administrations. Although the initial biodistributions were different, all routes resulted in specific tumor targeting. When using mice as an experimental model, it may be very convenient and provide a more consistent dose in some experimental plans to give the $\mathrm{mAb}$ SQ or IP. Finally, this is one of the first studies to conclusively demonstrate that the specific epitope bound by an antibody has significant therapeutic implications. This knowledge also may enhance development of synergistic drugs to be given with HB22.7.

Acknowledgments This work was supported in-part by Veterans Administration Merit Award, Leukemia and Lymphoma Society Translational Research Award, UC Davis Health Sciences Grant, the deLeuze Endowment for the nontoxic cure of lymphoma, and the Schwedler Family Foundation.

Conflict of interest statement The authors report no competing financial interests.

Open Access This article is distributed under the terms of the Creative Commons Attribution Noncommercial License which permits any noncommercial use, distribution, and reproduction in any medium, provided the original author(s) and source are credited.

\section{References}

1. Molina A (2008) A decade of rituximab: improving survival outcomes in non-Hodgkin's lymphoma. Ann Rev Med 59:237-250

2. Sato S, Tuscano JM, Inaoki M, Tedder TF (1998) CD22 negatively, positively regulates signal transduction through the $\mathrm{B}$ lymphocyte antigen receptor. Semin Immunol 10(4):287-297

3. Williamson JM, Grigor I, Smith ME et al (1987) Ploidy, proliferative activity, cluster differentiation antigen expression, clinical remission in high-grade non-Hodgkin's lymphoma. Histopathology 11(10):1043-1054

4. Law CL, Aruffo A, Chandran KA, Doty RT (1995) Ig domains 1, 2 of murine CD22 constitute the ligand-binding domain, bind multiple sialylated ligands expressed on B, T cells. J Immunol 155(7):3368-3376

5. Tedder TF, Tuscano J, Sato S, Kehrl JH (1997) CD22, a B lymphocyte-specific adhesion molecule that regulates antigen receptor signaling. Annu Rev Immunol 15:481-504

6. Engel P, Wagner N, Miller AS, Tedder TF (1995) Identification of the ligand-binding domains of $\mathrm{CD} 22$, a member of the immunoglobulin superfamily that uniquely binds a sialic aciddependent ligand. J Exp Med 181(4):1581-1586

7. Poe JC, Fujimoto Y, Haesegawa M et al (2004) CD22 regulates B lymphocyte function in vivo through both ligand-dependent and ligand-independent mechanisms. Nat Immunol 5(4):1078-1087
8. Tuscano J, Engel P, Tedder TF, Kehrl JH (1996) Engagement of the adhesion receptor $\mathrm{CD} 22$ triggers a potent stimulatory signal for B cells and blocking CD22/CD22L interactions impairs T-cell proliferation. Blood 87(11):4723-4730

9. Tuscano JM, Riva A, Toscano SN, Tedder TF, Kehrl JH (1999) CD22 cross-linking generates B-cell antigen receptor-independent signals that activate the JNK/SAPK signaling cascade. Blood 94(4):1382-1392

10. Tuscano JM, O'Donnell RT, Miers LA et al (2003) The anti-CD22 ligand blocking antibody, HB22.7, has independent lymphomacidal properties, and augments the efficacy of ${ }^{90}$ Y-DOTA-peptideLym-1 in lymphoma xenografts. Blood 101:3641-3664

11. Leonard M, Coleman JP, Ketas JC et al (2003) Phase I/II trial of epratuzumab (humanized anti-CD22 antibody) in indolent nonHodgkin's lymphoma. J Clin Oncol 21(16):3051-3059

12. Carnahan J, Wang P, Kendall R et al (2003) Epratuzumab, a humanized monoclonal antibody targeting CD22: characterization of in vitro properties. Clin Cancer Res 9(10 pt 2):3982S-3990S

13. McCarthy DW, Shefer RE, Klinkowstein RE et al (1997) Efficient production of high specific activity $64 \mathrm{Cu}$ using a biomedical cyclotron. Nucl Med Biol 24:35-43

14. Meares CF, McCall MJ, Reardon DT, Goodwin DA, Diamanti CI, McTigue M (1984) Conjugation of antibodies with bifunctional chelating agents: isothiocyanate, bromoacetamide reagents, methods of analysis, subsequent addition of metal ions. Anal Biochem 142:68-78

15. Tai YC, Chatziioannou AF, Yang Y et al (2003) MicroPET II: design, development and initial performance of an improved microPET scanner for small-animal imaging. Phys Med Biol 48:1519-1537

16. Chatziioannou A, Tai YC, Doshi N, Cherry SR (2001) Detector development for microPET II: a 1 micron resolution PET scanner for small animal imaging. Phys Med Biol 46:2899-2919

17. John B, Herrin BR, Raman C, et al. (2003) The B cell co-receptor CD22 associates with AP50, a clathrin-coated pit adapter protein, via tyrosine-dependent interaction. J Immunol. 170(7):3534-43

18. Phelps ME (2000) Inaugural article: positron emission tomography provides molecular imaging of biological processes. Proc Natl Acad Sci USA 97:9226-9233

19. Winnard P Jr, Raman V (2003) Real time non-invasive imaging of receptor-ligand interactions in vivo. J Cell Biochem 90:454-463

20. Chatziioannou A, Qi J, Moore A et al (2000) Comparison of 3-D maximum a posteriori, filtered back projection algorithms for high-resolution animal imaging with microPET. IEEE Trans Med Imaging 19:507-512

21. Haas KM, Sen S, Sanfrod IG et al (2006) CD22 ligand binding regulates normal, malignant $\mathrm{B}$ lymphocyte survival in vivo. J Immunol 177(5):3063-3073

22. Poe JC, Fujimoto Y, Hasegawa M et al (2004) CD22 regulates B lymphocyte function in vivo through both ligand-dependent, ligand independent mechanisms. Nat Immunol 5(10):1078-1087

23. Gordon LN, Grow WB, Pusateri A, et al. Phase II trial of individualized rituximab dosing for patients with CD20-postive lymphoproliferative disorders. 2005; 23(6):1096-102

24. Hale G, Rebello P, Brettman LR et al (2004) Blood concentrations of alemtuzumab, antiglobin responses in patients with chronic lymphocytic leukemia following intravenous or subcutaneous routes of administration. Blood 104:948-955 